\title{
Is Home Respiration effective?
}

Bei einem pneumologischen Meeting über die Atempumpe im Jahre 1987 in Göttingen kristallisierte sich heraus, dass die intermittierende Beatmung eine effektive Therapie der ventilatorischen Insuffizienz ist [1]. Die Theorie, dass durch die intermittierende Ruhigstellung der Inspirationsmuskulatur die erschöpfungsbedingten Funktionseinschränkungen durch Erholung beseitigt werden, so dass bei Spontanatmung die Atemmuskulatur funktionstüchtiger ist, ist bisher als Ursache der Verbesserung der arteriellen Blutgasspannungen nie zweifelsfrei bewiesen worden; sie hat sich aber als Modellvorstellung bis heute uneingeschränkt bewährt.

D. Robert berichtete über Patienten mit stabilen neuromuskulären Erkrankungen, die bei einer Beatmungszeit von 15/24 Std. eine 5-jährige Überlebenszeit von 95\% und eine 10-jährige Überlebenszeit von $87 \%$ aufwiesen. Demgegenüber waren die Ergebnisse der Heimbeatmung für Patienten mit COPD mit Überlebensraten von $18 \%$ über 5 Jahre sehr enttäuschend, was aber natürlich daran liegt, dass die Atemmuskelinsuffizienz bei Patienten mit COPD nur eines der Vielzahl der respiratorischen Probleme darstellt, während bei Patienten mit neuromuskulärer Erkrankung die Lunge annähernd gesund ist. Diese Ergebnisse der Heimbeatmung waren bei tracheotomierten Patienten gewonnen worden und D. Robert stellte bei diesem Meeting eine Alternative zur Tracheotomie vor: Die nichtinvasive Beatmung über individuell angefertigte Nasenmasken, die in Lyon seit 1985 entwickelt wurden. Da nun die Heimbeatmung als effektive Therapie der Atempumpinsuffizienz nichtinvasiv erfolgte, wurde die Indikation richtigerweise schon frühzeitig bei beginnender Hyperkapnie gestellt, damit lebensbedrohliche respiratorische Komplikationen erst gar nicht eintreten.
Die Ausweitung der Indikationen in ein möglichst frühes Stadium der Erkrankung erforderte natürlich den Wirksamkeitsnachweis der Heimbeatmung. Ein wichtiges Therapiekriterium ist die Prognoseverbesserung. Für Patienten mit thorakal-restriktiven und neuromuskulären Erkrankungen wird aber eine Prognoseverbesserung in kontrollierten Studien wahrscheinlich nie bewiesen werden, da alle Experten einheitlich von der hohen Effektivität der Heimbeatmung so überzeugt sind, dass sich kontrollierte Studien aus ethischen Gründen verbieten. Aus den Daten von D. Robert ging aber schon hervor, dass die Prognose von Patienten mit COPD deutlich schlechter ist, so dass in den letzten Jahren kontrollierte, randomisierte Studien begonnen wurden, um die Prognose bei Sauerstofflangzeittherapie und Heimbeatmung zu vergleichen. Ganz aktuell ist eine erste Studie publiziert, die bei 2-jähriger Beobachtung keine unterschiedliche Prognose ergab, wobei allerdings die Beatmungsmethode sehr kritisch zu beurteilen ist [2].

Neben der Lebensverlängerung hat aber die Verbesserung der Lebensqualität eine entscheidende Bedeutung bei der Beurteilung des medizinischen Therapieerfolges. Eine Lebensverlängerung bei gleichzeitiger Minderung der Lebensqualität erscheint oft als Horrorvision, wie z.B. ungewollte Lebensverlängerung durch medizinische Maßnahmen bei schwerkranken Patienten ohne Aussicht auf Verbesserung ihres Leidens. So kommentierte der stets allen Innovationen aufgeschlossene D. Nolte anlässlich der Publikation unserer ersten Ergebnisse über Weaning und Heimbeatmung 1991 in einem Editorial der Med. Klinik, dass es „sehr sorgfältig zu überlegen sei, ob wir ihn (den Patienten) wirklich unbedingt auf Dauer in die Abhängigkeit eines Beatmungsgerätes bringen müssen“ [3]. Hinter dieser Formulierung war die Besorgnis spürbar, dass wir ohnehin schwerbehinderten Menschen, die zum Teil schon rollstuhlabhängig sind, durch die 
Heimbeatmung eine weitere Bürde auferlegen und damit deren Lebensqualität noch stärker beeinträchtigen würden. Auch wir hatten anfänglich diese Befürchtungen, die wir in unserer Arbeitsgemeinschaft Heimbeatmung und Respiratorentwöhnung diskutierten. Unvergessen bleibt dabei die engagierte Argumentation eines Betroffenen mit schwersten Behinderungen und auf wenige Stunden limitierte Spontanatmung, dass gesunde Menschen sich ohnehin nicht in seine Lage versetzen könnten und daher seine Lebensqualität überhaupt nicht ermessen könnten. Die Ärzte hätten die Pflicht, die Betroffenen über alle Möglichkeiten der Behandlung aufzuklären und diese würden dann entscheiden, welche Therapieoptionen für sie infrage kämen. Dies sei ihr Recht auf selbstbestimmtes Leben und sie könnten diese Beatmungsoption ausprobieren und dann entscheiden, ob sie weitergeführt wird oder nicht. Dieser Argumentation konnten wir uns gut anschließen, zumal nach der oben beschriebenen $\mathrm{Er}$ holungstheorie die intermittierende Beatmung niemand in die Abhängigkeit eines Beatmungsgerätes bringt, sondern durch die erholungsbedingte Stärkung der Atemmuskelfunktion die Atmung erleichtert. Ein Abbruch der Beatmung führt nicht zu einer Verschlechterung der Erkrankung, sondern lediglich zu dem Status quo ante. Tatsächlich lehnen bei korrekter Indikationsstellung und optimaler Maskenanpassung und Beatmungseinstellung nur wenige Patienten die Heimbeatmung nach einer Probephase von 6-12 Wochen ab. Einige Patienten profitieren in ihrer Lebensqualität derart, dass sie trotz Rollstuhlabhängigkeit und intermittierender Beatmung weite Reisen in andere Kontinente unternehmen, z.T. Sport treiben können und wieder berufstätig werden.

Um aber subjektive Erfahrungen in klinischen Studien zu belegen, muss die Lebensqualität messbar gemacht werden. Die messbare gesundheitsbezogene Lebensqualität ist ein psychologisches Konstrukt, dass die subjektive Befindlichkeit eines Patienten unter Berücksichtigung physischer, psychischer, sozialer und funktioneller Aspekte charakterisiert. Die subjektive Wahrnehmung des Patienten wird dabei von der Grunderkankung, vom Verlauf und auch von der Behandlung beeinflusst. Bei der Erfassung der Lebensqualität steht die eigene subjektiv empfundene Befindlichkeit des Patienten im Vordergrund: Der Patient und nicht das medizinische Personal oder Angehörige entscheidet über das Ausmaß evtl. Einschränkungen. Bei der Erfassung der gesundheitsbezogenen Lebensqualität dominieren Fragebögen, die der Patient beantwortet. Leider existiert keiner, mit dem - unabhängig von der Erkrankung - alle Aspekte der Lebensqualität bewertet werden können. So gibt es Fragebögen, die einen Vergleich zwischen verschiedenen Patientenkollektiven und einem Normalkollektiv erlauben (krankheitsübergreifend) und andere, die die therapiebedingten Veränderungen der Lebensqualität erfassen können (krankheitsspezifisch).
In diesem Heft geben W. Windisch und Mitarbeiter einen Überblick über die Problematik der Messung von Lebensqualität und stellen unter Einbeziehung eigener Untersuchungen die bisherigen Ergebnisse bei heimbeatmeten Patienten vor [4]. Beruhigend ist, dass sich die psychische Lebensqualität bei heimbeatmeten Patienten, trotz schlechterer körperlicher Lebensqualität, in keinster Weise von der psychischen Lebensqualität von Patienten mit chronischen Lungenerkrankungen unterscheidet. Es existieren sogar Gruppen mit starker körperlicher Einschränkung, bei denen die psychische Lebensqualität gegenüber einem Normalkollektiv gleich bzw. sogar besser ist! Aus der Übersicht geht aber auch eindeutig hervor, dass die methodische Weiterentwicklung geeigneter, krankheitsspezifischer Fragebögen ein wichtiges Ziel ist, wenn in Zukunft Änderungen der Lebensqualität im Zuge der Heimbeatmung sicher beurteilt werden sollen. Dies geht auch aus der oben erwähnten Studie bei Patienten mit COPD hervor, bei der erst die Modifikation von standardisierten Fragebögen eine Besserung der Lebensqualität aufzeigte [2].

Von den Autoren wurde daher erstmals ein krankheitsspezifischer Fragebogen, der überhaupt für diese Fragestellung geeignet ist, entwickelt und validiert [4]. Dieser Fragebogen ist Kernstück einer aktuell initiierten Multicenter-Studie, die das Ziel hat, Änderungen der Lebensqualität nach Einleitung der Heimbeatmung prospektiv zu erfassen.

Solange diese Ergebnisse jedoch noch nicht vorliegen, müssen wir bei unserem seit 15 Jahren bewährtem Verfahren bleiben: Nach Indikationsstellung wird eine mehrwöchige intermittierende Beatmung durchgeführt und nach einer Kontrolluntersuchung nur dann fortgeführt, wenn sich das subjektive Befinden und die Blutgasspannungen bei Spontanatmung deutlich gebessert haben. Dabei darf die subjektive Bewertung des Therapieerfolges der objektiven Bewertung in keiner Weise nachstehen. Hier wird in der Übersicht von W. Windisch und Mitarbeitern deutlich, wie wichtig es ist, die sensitiven, vom Patienten erlebten Kriterien des Therapieerfolges von den objektiven Funktionsparametern zu trennen. Auf die Studienergebnisse der nächsten Jahre darf man somit sehr gespannt sein.

\section{Literatur}

${ }^{1}$ Robert D, Laier-Groeneveld G, Leger P. Mechanical Assistance. In: Criée C-P, Hüttemann U: The Respiratory Pump/Die Atempumpe. Prax Klin Pneumol 1988; 42: 846 - 849

2 Clini E, Sturani C, Rossi A, Vlaggi S et al. The Italian multicentre study on noninvasive ventilation in chronic obstructive pulmonary disease patients. Eur Respir J 2002; 20: 529-538

${ }^{3}$ Nolte D. Soll sich ein Patient selbst beatmen? Med Klin 1991; 86: 269-270

${ }^{4}$ Windisch W, Freidel K, Matthys H, Petermann F. Gesundheitsbezogene Lebensqualität bei Patienten mit Heimbeatmung. Pneumologie 2002; 56: $610-620$ 